\title{
Mussolini e i capi provincia della RSI
}

Rovatti

ISSN: 2282-4979

DOI: 10.12977/ere-

view262

Il saggio analizza il metodo di lavoro di Mussolini e il rapporto tra il "centro" e la "Periferia" della Repubblica sociale italiana attraverso il rapporto tra Mussolini e i suoi prefetti. Lo scopo è di capire non solo come funzionava il governo della RSI, ma anche la responsabilità di Mussolini nel processo di brutalizzazione della guerra civile.

The essay analyzes the working method of Mussolini and his relationship between the "center" and the "periphery" of the Italian Social Republic through the connection between Mussolini and his prefects. The aim is to understand not only how the govern of the RSI worked, but also the responsibility of the Dictator in the brutalization of the Italian civil war.

La Repubblica sociale italiana ha rappresentato, per oltre seicento giorni, il governo di fatto di una parte importante del territorio nazionale. Nonostante le difficoltà, la Repubblica dovette gestire l'amministrazione, riscosse tasse, controllò la produzione e la distribuzione dei beni primari, distribuì la posta, tentò di mantenere in funzione i trasporti pubblici. Era ai funzionari e agli uffici locali della Repubblica che i cittadini dovevano rivolgersi per le loro pratiche burocratiche quotidiane, era l'amministrazione fascista che faceva sì che la vita civile potesse ancora continuare. Anche se coinvolta nella guerra civile, la Rsi non fu soltanto repressione, e in una sua storia complessiva bisognerebbe analizzare a fondo, e a livello sia locale che generale, molti altri aspetti. Ad esempio il controllo della produzione e della distribuzione dei generi alimentari, che assillava i capi provincia almeno quanto la Resistenza, oppure il tema dei rapporti con i tedeschi, o ancora la gestione degli sfollati. Tutte questioni che videro i capi provincia in prima 
linea e che avevano un significato politico, oltre che economico e amministrativo, di enorme importanza per la vita quotidiana dei cittadini a loro affidati. Prospettive tuttavia troppo estese per essere affrontate in un unico saggio, che analizzerà invece proprio il tema della repressione per capire quale fosse il rapporto tra il centro della Rsi, ovvero Mussolini, e i rappresentanti locali del potere, ovvero i capi provincia, e quale fosse il grado di autonomia di questi ultimi.

\section{Il centro del potere: la scrivania del Duce}

Secondo le memorie di alcuni ex fascisti repubblicani Mussolini a Salò sarebbe diventato un uomo senza reali interessi per la politica ed il governo, privo non solo di potere, saldamente nelle mani dei tedeschi, ma anche di iniziativa. L'oramai periclitante dittatore sarebbe divenuto, secondo la memorialistica (assunta poi da parte della storiografia), quasi un filosofo, impegnato in riflessioni sulla storia d'Italia e del fascismo, piuttosto che un uomo d'azione, qual era sempre stato'.

Eppure, analizzando il suo calendario di lavoro, si nota immediatamente come, nonostante tutte le difficoltà, Mussolini avesse tentato di mantenere intatto il suo stile di vita e soprattutto la sua prassi lavorativa, pur non riuscendo più, per ovvi motivi, a mantenere quell'ordine e quella disciplina che avevano contraddistinto la sua giornata tipica degli anni "belli" del regime?2.

La giornata lavorativa mussoliniana, dal 1923 al 1943, rimase sostanzialmente sempre uguale. Soprattutto dal 1930, anno del trasferimento degli uffici personali del duce da palazzo Chigi a palazzo Venezia, l'agenda degli impegni rimase più o meno la stessa. Se non impegnato in viaggi, oppure in manifestazioni entro la capitale, Mussolini si recava in ufficio tra le otto e mezza e le nove, dove rimaneva fino all'ora di pranzo. Dopo una sosta di un paio d'ore a casa, a villa Torlonia, rientrava in ufficio verso le tre e mezza e vi rimaneva, a seconda dei giorni, fino alle sette/otto di sera.

Le udienze mattutine erano dedicate ai suoi più importanti collaboratori. Così i primi ad essere accolti erano i comandanti di carabinieri, polizia e l'ufficio speciale riservato, la struttura che intercettava le telefonate. Dopo questo primo giro, 
venivano ricevuti i ministri o i sottosegretari dei ministeri più importanti. Nel pomeriggio venivano invece ricevuti i visitatori esteri, giornalisti, capi di Stato e diplomatici, i visitatori privati e più raramente delegazioni collettive, nonché le sue amanti.

La mattina era il momento più importante della giornata. I suoi collaboratori si presentavano a rapporto, esponevano problemi e suggerivano soluzioni, a cui Mussolini rispondeva con indicazioni che, molto spesso, rispecchiavano la volontà e l'iniziativa del sottoposto. Tenendo conto dell'enorme numero di cariche ricoperte dal dittatore, infatti, e dall'infinito numero di pratiche che gli venivano sottoposte, Mussolini non aveva il tempo per approfondire o analizzare con la necessaria attenzione tutti i provvedimenti che gli venivano presentati, con il risultato che firmava praticamente tutto quello che gli veniva proposto.

In pratica, Mussolini passava l'intera giornata lavorativa a palazzo Venezia che, negli anni Trenta, era diventato il vero centro, reale e simbolico, del regime fascista. Chi aveva accesso all'ufficio privato del dittatore, e poteva vantare una vicinanza anche fisica con Mussolini, aveva anche un maggiore peso all'interno delle gerarchie fasciste, a prescindere dal ruolo ufficiale. In pratica si era instaurato un rapporto di tipo feudale, secondo la definizione di Kershaw ${ }^{3}$, tra Mussolini e i suoi più fedeli collaboratori, i quali venivano di volta in volta riconfermati nel loro ruolo e nella loro importanza grazie all'udienza concessa dal duce. Lo stesso valeva anche per i gerarchi di minore importanza. Per un ras locale, una visita a palazzo Venezia, magari coronata dalla notizia sulla stampa ${ }^{4}$, rivelava al volgo il rango raggiunto dal fortunato che «aveva parlato con il duce», rinforzandone quindi la statura nell'ambito della politica locale.

Nei momenti più difficili e tragici della fine della guerra, il ritmo di lavoro divenne frenetico, come ad esempio alla fine della guerra. Non fidandosi evidentemente più di nessuno Mussolini, nel disperato tentativo di mantenere in piedi un paese e un governo ormai al collasso, continuava a ricevere persone nella vana speranza di controllare e risolvere problemi oramai irrisolvibili. Nell'estate del 1943 era arrivato a ricevere fino a 30 persone nella stessa giornata, che andavano dai vertici militari a singoli visitatori, i quali apparentemente non avevano alcun ruolo preciso o potevano fornire alcun aiuto nella condotta della guerra, sintomo

Kershaw 2000, p. 278.

Sul "Popolo d'Italia" esisteva una apposita rubrica intitolata "Le udienze del duce". Un tipico esempio è quello del 15 luglio 1938: «ll Duce ha pure ricevuto lo scrittore Ivon De Begnac, col quale si è intrattenuto su questioni di carattere giornalistico». 
abbastanza evidente che il dittatore aveva oramai perso completamente il controllo della situazione e della sua stessa giornata lavorativa.

Durante la Repubblica sociale italiana il sistema di governo rimase, sostanzialmente, lo stesso. Tra i primi atti di Mussolini ci fu quello di ricostituire la Segreteria particolare del duce, l'organismo che sovrintendeva e regolava il sistema delle udienze. Dopo un primo periodo piuttosto caotico, durato fino all'inizio di dicembre 1943, il sistema tornò a funzionare in maniera abbastanza ordinata, sotto la direzione prima di Giovanni Dolfin e poi di Ugo Cellai.

Secondo il colonnello Johann Jandl, ufficiale di collegamento con la Wehrmacht:

Il Duce fa un'impressione migliore che non al tempo del mio primo rapporto. In particolare, la sua voglia di lavorare e la quantità di lavoro che riesce a svolgere ogni giorno sono notevolmente aumentati. Ora va regolarmente in ufficio alle 8,45 , e riceve subito i visitatori sino alle $2-2,30$. Fa una breve pausa di circa mezz'ora a mezzogiorno e nel pomeriggio continua a lavorare di solito sino alle 9. Spesso lavora di notte per conto proprio [Deakin 1963, 599].

Anche a Gargnano, Mussolini riceveva nella mattinata i responsabili della repressione e delle forze armate - Buffarini Guidi, Graziani e Pavolini tra tutti - e nel pomeriggio gli altri postulanti, tra i quali capi provincia, ras locali, ufficiali dell'esercito, giornalisti e privati cittadini. Ovviamente, nel corso della giornata, erano numerosi anche i tedeschi. Ogni giorno veniva ricevuto un ufficiale di collegamento con la Wehrmacht e spesso anche i proconsoli del Reich, l'ambasciatore Rudolph Rahn e l'alto comandante della polizia e delle SS Karl Wolff. Questo, ad esempio, il calendario delle udienze di venerdì 29 marzo $1944^{5}$.

\begin{tabular}{|l|l|}
\hline Presidenza & \\
\hline Esteri & $\mathrm{x}$ \\
\hline Partito & \\
\hline Interno & \\
\hline Cultura Popolare & $\mathrm{x}$ \\
\hline Pancino & \\
\hline Slataper & \\
\hline Prefetto Dinale & \\
\hline Ridolfi & $\mathrm{x}$ \\
\hline Ministro Tarchi & 10.00 \\
\hline
\end{tabular}

DDHI. I nomi riportati in corsivo sono quelli che nell'originale sono scritti a mano. La X accanto a dei nomi, sempre manoscritta, veniva messa di pugno da Mussolini. 


\begin{tabular}{|l|l|}
\hline Tassinari & $\mathrm{x}$ \\
\hline Sig.ra Pallottelli & $\mathrm{x}$ \\
\hline Farinacci & $\mathrm{x}$ \\
\hline Redattori Crociata Italica & $\mathrm{x}$ \\
\hline Col. Jandl & $\mathrm{x}$ \\
\hline Presidenza & 16.00 \\
\hline Interno & 16.15 \\
\hline Partito & 16.30 \\
\hline Prefetto Dinale & 17.00 \\
\hline Padre Pancino & 17.30 \\
\hline Col. Guido Slataper & 18.00 \\
\hline Ridolfi & $\mathrm{x}$ \\
\hline Prof. Vikoler & \\
\hline
\end{tabular}

Tra i ministri che più spesso avevano accesso allo studio di Mussolini vi erano il sottosegretario alla Presidenza del consiglio Francesco Maria Barracu e il ministro degli Esteri Serafino Mazzolini.

Esaminando però più a fondo il calendario delle udienze, si nota una differenza fondamentale rispetto agli anni del regime, dovuta al sistema statuale caotico e frammentato della Repubblica sociale. Le udienze del periodo repubblicano, infatti, registrano una frequenza molto maggiore delle autorità locali, tra le quali i capi provincia, ma anche di personaggi come i comandanti delle varie "bande" e gruppi autonomi che infestarono il territorio della Rsi per tutta la sua breve esistenza. Come si spiega questa contraddizione? Qual era il motivo che portava Mussolini a interessarsi così intensamente dei problemi delle province? E chi comandava nei territori locali? I prefetti o i nuovi ras locali?

All'inizio dell'autunno del 1943 l'amministrazione statale era andata praticamente in frantumi, e con essa anche il sistema partito. Nelle città e nelle province non erano più i prefetti o i federali, organizzati e controllati dal Ministero dell'Interno o dalla Segreteria nazionale del Pnf, a governare, ma erano capi locali, spesso improvvisati, che erano riusciti a rimettere le mani sul potere, approfittando del vuoto causato dal caos post armistiziale o della loro vicinanza con gli occupanti tedeschi. Esempi classici di questa situazione sono i vari triumvirati che avevano riaperto alcune federazioni, come a Bologna, dove lo scontro interno aveva portato quasi al collasso della Federazione. Un altro esempio eclatante è Roma, dove uno sconosciuto funzionario della Federazione dei commercianti, Gino Bardi, e uno squadrista della prima ora emarginato durante il regime, Guglielmo Pollastri- 
ni, avevano riaperto e gestito, sia pure per un breve periodo, la Federazione più importante d'Italia. Altro centro di potere locale erano le decine di reparti autonomi di polizia o delle forze armate, che si stavano ricostituendo sulle ceneri della Milizia e del Regio esercito. Basti pensare a reparti notissimi come la X Flottiglia Mas, nata da una iniziativa personale del comandante Junio Valerio Borghese, o la Legione autonoma Ettore Muti, comandata da uno spostato come Franco Colombo, ma anche a reparti meno noti ma altrettanto potenti, almeno a livello locale, come la "Sicherai" [Sicherheit] di Felice Fiorentini nell'Oltrepò pavese ${ }^{6}$. Insomma un insieme di centri di potere locale che avevano spesso un rapporto assai labile con il "centro" e con il governo, e alle volte dovevano la loro potenza e la loro influenza al rapporto con i tedeschi. Tuttavia con la stabilizzazione della Rsi, alla fine del 1943, Mussolini tentò di rimettere in piedi una amministrazione degna di questo nome, e per farlo si affidò, come durante il regime, ai prefetti, ora rinominati capi provincia. Il cambiamento del nome aveva un significato preciso: la nuova figura doveva essere il dominus della situazione locale, una istituzione di riferimento per qualsiasi problema interno alla provincia, superiore alle altre autorità politiche e militari. Fu una delle prime decisioni prese da Mussolini, che il 27 settembre 1943 convocò il governo alla Rocca delle Caminate per informare i ministri della nuova denominazione e dei compiti di questi funzionari ${ }^{7}$. Anche la frequenza con la quale Mussolini ricevette i suoi capi provincia, dimostra l'importanza data a tali figure. Tra l'ottobre del 1943 e il febbraio del 1945, le udienze a loro riservate furono oltre 100, mentre alcuni di essi, come ad esempio Piero Parini, furono ricevuti più volte 8

Trovare il personale per gestire una situazione così caotica, e ripristinare una amministrazione degna di questo nome, non fu una cosa facile, come si evince dal notevole turn over degli stessi capi provincia. Come si vede dall'elenco qui di seguito, in alcune città si arrivò a cambiare fino a quattro prefetti nell'arco dei "seicento giorni", cosa che dimostra quanto fosse difficile gestire il potere nelle varie periferie e quanto fosse altrettanto difficile trovare uomini sufficientemente

\footnotetext{
Un elenco di molte di queste "bande" è deducibile da: Franzinelli 2018. I libro di Mimmo Franzinelli, Tortura. Storie dell'occupazione nazista e della guerra civile, Mondadori, Milano, 2018.

«Dopo lunghe attese al campo di Ciampino ed all'Ambasciata germanica a mezzogiorno partiamo in automobile alla volta della Rocca delle Caminate. Ci sono Graziani, Gai, Mezzasoma, Pellegrini Giampietro, Barracu, Biggini. Dopo una sosta a Spoleto per la colazione arriviamo a Forlì sotto la pioggia torrenziale alle 20. Ci aspetta Buffarini Guidi. E con lui ascendiamo alla Rocca. II Duce riceve i membri del Governo ai quali legge la dichiarazione che preannuncia la Costituente, scioglie il Senato, unifica le Confederazioni, definisce la figura del Capo della Provincia» [Rossi 2005, 340].
} 
validi, e soprattutto non corrotti, che potessero rappresentare localmente il potere centrale.

Ad esempio ad Alessandria si alternarono Giambattista Alessandri e Mario Piazzesi; ad Aosta Cesare Augusto Carnazzi e Bruno Stefanini; ad Asti Ancora Carnazzi, Renato Celio e Paolo Quarantotto; a Bergamo Emilio Grazioli e Rodolfo Vecchini; a Bologna Alberto Zaccherini, Dino Fantozzi e Guglielmo Montani; a Ferrara Giuseppe Altini, Vincenzo Berti e Enrico Vezzalini; a Genova Carlo Emanule Basile, Arturo Bigoni e Guido Letta; a La Spezia Giovanni Appiani, Manlio Binna, e Francesco Turchi; a Livorno Edoardo Facduelle e Giannino Romualdi; a Milano Mario Bassi, Piero Parini, Carlo Riva e Oscar Uccelli; a Novara Gaspero Barbera, Dante Maria Tuninetti, Alberto Zaccherini e di nuovo Vezzalini; a Piacenza Davide Fossa, Alberto Graziani e Mario Piazzesi; a Pistoia Emilio Balletti, Antonio Cocchi e Giuseppe Giovine; a Torino Emilio Grazioli, Edoardo Salerno e Paolo Zerbino; a Venezia Gaspero Barbera, Dino Cagetti e Piero Cosmin; a Vicenza Neos Dinale, Edgardo Preti e Filippo Mirabelli.

\section{Uomini comuni? I capi provincia della Rsi}

Secondo Luigi Ganapini, che riprende in parte i giudizi della memorialistica fascista, i motivi della scelta e la prassi di governo dei funzionari che aderirono alla Rsi si estrinsecarono anche «come attività amministrativa, nell'esplicazione di compiti legati alla vita delle comunità, resi urgenti e cruciali dalla congiuntura bellica» [Ganapini 1999, 254]. Oltre agli “idealisti”, secondo Ganapini, governarono le province anche dei funzionari che si assunsero un compito estremamente difficile per spirito di servizio, per mantenere in piedi lo Stato e l'amministrazione. «È questo quindi il mondo di quanti scelgono Salò in nome del servizio allo Stato, del loro ruolo all'interno di esso, della stabilità del quotidiano mondo moderato: valori variamente dosati e distribuiti su tutte le sfumature dell'universo dell'ordine» [Ganapini 1999, 254]. I capi provincia, effettivamente, dovettero sobbarcarsi compiti estremamente difficili e, data la situazione, quasi impossibili: dovevano cercare di gestire non solo l'ordine pubblico ma, ad esempio, la distribuzione dei generi alimentari, del vestiario, vigilare sulla produzione agricola, reprimere la borsa nera, trattare con i tedeschi, prendersi cura di migliaia di sfollati provenienti dal sud e dalle città bombardate, eccetera. Inoltre le difficoltà erano aumentate non solo dalla invadenza dell'alleato occupante ${ }^{9}$, dalla distruzione di 
uffici ed archivi, dalle difficoltà delle comunicazioni, ma anche dalla scarsa affidabilità del personale a disposizione. Moltissimi funzionari pubblici si erano dati alla macchia nel settembre del 1943, molti altri, pur rimanendo al loro posto si limitavano ad andare in ufficio cercando di evitare di compromettersi troppo con una Repubblica dal futuro troppo incerto. Altri ancora facevano invece il doppio gioco, passando informazioni alla Resistenza e boicottando la Rsi ${ }^{10}$.

Compiti e situazioni estremamente difficili che spesso non avevano una soluzione. Alcuni, come Piero Parini, tentarono la carta del "patriottismo" locale, ovvero cancellando quasi ogni accenno al fascismo e alla repubblica, per far leva invece sulle necessità della zona e sul senso di appartenenza cittadino o provinciale. Questi funzionari, in genere, tentarono di evitare di inasprire la guerra civile con violenze considerate inutili, come le rappresaglie, e di fare della "buona amministrazione". Altri invece, soprattutto se non provenivano dalla zona, si dimostrarono estremamente brutali anche nei confronti della popolazione civile, applicando i diktat nazisti o le direttive di Mussolini con ferocia.

Chi erano dunque gli uomini a cui Mussolini affidò il governo delle province? Si trattò di persone che gestirono «l'ordinaria amministrazione», secondo la definizione di Matteo Stefanori?" Tra loro vi erano anche dei funzionari legati più allo Stato e alle loro comunità, secondo quanto sostenuto da Ganapini? Si trattò di "uomini comuni", nell'accezione di Christopher Browning? Oppure furono dei fascisti "hard core", fedelissimi della prima ora, pronti a tutto per difendere il fascismo e il suo capo?

Premesso che allo stato delle ricerche è impossibile tentare una biografia collettiva completa dei tutti i prefetti della Rsi, alcuni dei quali risultano ancora dei perfetti sconosciuti, tuttavia attraverso le carriere di alcuni di essi è possibile proporre almeno una prima ipotesi di ricerca sulle esperienze, l'ideologia e la mentalità di un gruppo di funzionari che rimasero, fino alla fine, agli ordini di Mussolini. Emilio Grazioli, nato nel 1899, volontario nella Prima guerra mondiale a soli diciassette anni, era stato legionario fiumano per poi continuare la carriera all'interno del fascismo triestino, uno dei luoghi dove il movimento era stato più brutale e sicuramente il più razzista d'Italia. Ufficiale della Milizia dal 1923 al 1936 nel Carso, volontario in Africa Orientale e poi federale prima di Trento (1936) e poi

Per avere un'idea delle dimensioni del fenomeno, basta vedere il fondo Acs, Ministero dell'Interno, Divisione del personale, Personale fuori servizio. Qui sono conservati i fascicoli personali dei funzionari di Polizia. Anche se molte delle informazioni sul "doppio gioco" date dai funzionari stessi dopo la guerra sono probabilmente gonfiate per evitare l'epurazione, il fenomeno risulta incredibilmente esteso. 
di Trieste (1936-1939) [Savino 1937, 479]. Esponente del gruppo farinacciano [Lupo 2000, 396], venne messo proprio sul Carso dove, con la Milizia, doveva reprimere l'irredentismo sloveno. Sempre a Trieste, la campagna antisemita avrà i più entusiasti proseliti a partire dal 1938 .

Nel 1941 Grazioli venne nominato Alto commissario per la neo annessa Provincia italiana di Lubiana, un ruolo estremamente difficile dove alternò una politica relativamente "aperta" nei confronti della cultura slovena, ad una strategia estremamente dura nei confronti della stessa popolazione, colpevole di appoggiare la Resistenza. Assieme ai vertici militari, Grazioli reagì in maniera violentissima ad ogni attacco partigiano, permettendo fucilazioni di massa di ostaggi e tentando, senza riuscirci, di gestire in proprio, con le sole forze di polizia, di stroncare $i$ nuclei partigiani attestati sul monte Krim, il massiccio che sovrasta la città di Lubiana'.

Durante la Rsi, dopo essere stato capo provincia di Bergamo e di Ravenna, nell'ottobre del 1944 assunse la stessa carica a Torino. Nel febbraio del 1945 Mussolini lo nominò Alto commissario per il Piemonte. Lo spostamento in quest'ultima regione di Grazioli in sostituzione dello sbiadito Edoardo Salerno, già Capo della provincia di Roma, si può capire se si tiene conto del tracollo della Rsi in Piemonte. Dopo il fallimento del tentativo di riconquistare le montagne attraverso il rastrellamento organizzato l'estate precedente con la "Marcia contro la Vandea", probabilmente Mussolini ebbe intenzione di giocare le sue ultime carte mandando nella "Vandea badogliana" un personaggio esperto in repressione e antiguerriglia. Anche se oramai da mesi la violenza aveva subito una escalation, l'ultimo inverno della guerra civile in Piemonte fu particolarmente sanguinoso. In questo quadro di guerra ormai aperta e quotidiana, Grazioli diede un suo importante contributo alla brutalizzazione del fascismo. Scrive Nicola Adduci:

L'estrema rigidità di Grazioli e lo zelo [del questore] Protani vogliono essere il segnale di un cambiamento di passo e non solo una reazione alla perdita di prestigio e alla «debolezza» mostrate in precedenza da Salerno. L'obiettivo del nuovo corso sembra essere ora la riaffermazione del primato dello Stato sul partito per poter recuperare la piena autonomia nell'azione verso la comunità; paradossalmente, però, la strategia utilizzata per recuperare autorevolezza agli occhi del Pfr - Bn passa attraverso azioni di rappresaglia che colpiscono la componente operaia della comunità e si rivelano dunque decisamente controproducenti [Adduci 2014, 285].

L'azione era sicuramente controproducente, ma era perfettamente nella logica non solo del fascismo, ma anche di un personaggio quale Grazioli, che aveva 
cominciato la sua carriera politica combattendo proprio contro gli operai durante gli anni della "rivoluzione", ovvero nel periodo antemarcia. C'è una coerenza di fondo in tutta la carriera di Grazioli, e una coerenza anche nella sua azione politica, che si radicalizzò mano a mano che le sue esperienze personali lo abituarono alla violenza, e che i nemici si facevano sempre più forti e pericolosi. Per tutta la sua vita Grazioli aveva combattuto contro slavi e comunisti, iniziando nel 1922 e continuando per tutto il periodo della guerra. In questo, Grazioli è tipico esponente della generazione che aveva combattuto una guerra civile a livello europeo. Carlo Emanuele Basile, nato nel 1885, aveva cominciato la sua carriera nel Partito liberale diventando sindaco di Stresa, il paese dove era cresciuto. Volontario nella Prima guerra mondiale, salì la tipica scala gerarchica all'interno del fascismo: segretario del fascio locale, console della Milizia dal 1928, volontario in Etiopia e in Spagna e deputato al Parlamento [Savino 1937, 479]. Nel settembre del 1943 Basile aderì alla Repubblica sociale, e gli venne affidato il delicato incarico di capo della provincia di Genova, una delle province più difficili e turbolente.

Fino al giugno del 1944, quando diventò sottosegretario alle Forze armate, gestì la sua provincia con pugno di ferro. Tra le tante operazioni da lui organizzate, vi fu la fucilazione di otto prigionieri politici uccisi il 14 gennaio 1944 per rappresaglia dopo un attentato dei Gap di Genova ${ }^{13}$. Fu Basile, infatti, a convocare il Tribunale straordinario che condannò a morte le vittime, completamente estranee ai fatti. Il fatto che più viene ricordato, tuttavia, fu la deportazione dei quasi 1500 operai genovesi prelevati dai tedeschi il 16 giugno 1944 quale risposta punitiva per punire agli scioperi delle grandi fabbriche metallurgiche. Anche in queste operazioni Basile diede il suo contributo minacciando gli operai prima sulla stampa [Mayda 202, 243], e poi collaborando con i nazisti nelle prime operazioni di rastrellamento. Il 28 giugno Basile venne promosso da Mussolini a sottosegretario per l'Esercito, mentre il suo posto venne preso da Arturo Bigoni, già questore di Genova, anche lui dimostratosi particolarmente zelante nel collaborare con i nazisti.

Enrico Vezzalini era nato a Rovigo nel 1904. Non si sa quasi nulla del suo curriculum precedente all'8 settembre. Secondo un anonimo del 1944:

È conosciutissimo, sia a Modena che in provincia, come un megalomane, fanfarone, prepotente e libertino. È inviso a tutta la popolazione per il suo modo di trattare e per le prepotenze commesse durante il periodo trascorso a Modena, in qualità di Vice-comandante federale della G.I.L. Prima della guerra di Spagna, esercitava, con scarsissimo successo (era conosciuto col nomignolo di «Avvocato delle cause 
perdute») la professione di avvocato. [...] Al suo ritorno [dalla Spagna] valendosi dell'amicizia del Federale Pagliani, diventò Vice Comandante della G.I.L. ${ }^{14}$

La sua carriera ebbe evidentemente una notevole accelerazione, se nel novembre del 1943 partecipò al Congresso di Verona. Fu lui, assieme ad altri complici, a recarsi a Ferrara e a massacrare le vittime della strage del Castello Estense, avvenuta il giorno successivo come rappresaglia per la morte di Igino Ghisellini, federale di quella città. Come si legge nella sentenza della Corte di Assise di Novara, che lo giudicò nel 1945: «il Vezzalini fu notato alla finestra del Castello ridere soddisfatto alla vista dei cadaveri»» ${ }^{15}$. In seguito, «in evidente premio di tale sua attività fu nominato commissario federale e poi capo della provincia di Ferrara». Qui si fece notare per la sua brutalità. Sotto il suo comando operò in provincia il malfamato reparto speciale conosciuto con il nome di "Tupin", una specie di squadrone della morte che aveva come scopo principale quello di commettere esecuzioni clandestine di partigiani e antifascisti [Balugani 1999]. Dal luglio 1944 Vezzalini, sempre seguito dai “Tupin”, diventò capo provincia di Novara dove, agli ordini del questore Pasqualy, operava una sezione speciale della Questura denominata "La Squadraccia".

Fu il Vezzalini - si legge sempre nella sentenza della Corte di Assise Straordinaria - infatti, che preparò l'ambiente violento fra i suoi dipendenti attraverso discorsi e fatti da lui stesso ordinati: fu egli a servirsi dei Tupin e della Squadraccia per la consumazione di crimini; a lui più volte si fece ricorso dagli interessati per trovare protezione, ed egli, anziché reprimerlo, esaltava l'operato dei suoi dipendenti ${ }^{16}$.

In una ulteriore sentenza di condanna dei componenti della "Squadraccia", si legge che la sua attività si concretizzava in

rastrellamenti, con conseguenti eventuali catture, sevizie ed uccisioni di partigiani, e rappresaglie nei confronti della popolazione civile, consistenti in incendi, saccheggi, uccisioni [e] prelievi di ostaggi, al che spesso, poi, per iniziativa di singoli o di gruppi, si univano rapine, estorsioni, furti ed altri fatti integranti reati contro il patrimonio e la pubblica amministrazione ${ }^{17}$.

Si sta parlando, insomma, di un funzionario di chiara estrazione politica, che utilizzò la sua carica con decisione non comune perfino nel quadro di violenza

\footnotetext{
14 Acs, Ministero dell'Interno Rsi, Gabinetto, b. 26.

15 II testo della sentenza è riprodotto in "leri Novara Oggi", 1996, 4-5, 166.

16 "Ieri Novara Oggi", 1996, 4-5, 168.

Ivi, 174-175. Pasqualy comandava personalmente i plotoni di esecuzione. Ad esempio il 14 agosto 1944 partecipò alla fucilazione di due partigiani al Poligono di tiro di Novara. Acs, Ministero dell'Interno, Carte SIS, Sezione II, b. 35.
} 
scatenatosi nella guerra civile italiana. E tuttavia si tratta allo stesso tempo di un funzionario che seppe meglio concretizzare la tanto sbandierata fusione delle funzioni di prefetto e segretario politico, tanto da raggiungere, almeno nel ferrarese, risultati amministrativi di un certo rilievo ${ }^{18}$. In controtendenza si può vedere il curriculum vitae di Edoardo Salerno, già capo provincia di Roma dove si era fatto notare pochissimo, un po' probabilmente per le oggettive difficoltà di gestire una città nel caos (con decine di migliaia di persone sfollate dalle provincie meridionali o in fuga dai bombardamenti, e con una situazione annonaria ormai in piena crisi), ma anche perché a comandare erano unicamente i tedeschi. Le sue esperienze precedenti rivelano il profilo di un funzionario di carriera, proveniente dal fascismo, ma che poi aveva abbracciato la carriera amministrativa. Era stato infatti federale di Catanzaro dal 1922 al 1924, per diventare deputato dal 1924 al 1929 e poi prefetto in varie città. Prima di reggere la Prefettura di Roma, era stato assegnato a Trapani, Siracusa e Brescia. Dopo la Prima guerra mondiale e la Marcia su Roma, non aveva più avuto esperienze di guerra ${ }^{19}$. Salerno era quindi un personaggio che si può definire un "funzionario comune", che probabilmente rimase al suo posto per senso del dovere e per lealtà nei confronti di Mussolini e del fascismo, ma che certo non diede prova di particolare efficienza né brutalità, che per i vertici della Rsi erano praticamente sinonimi. Durante i mesi dell'occupazione nazista della capitale, Salerno fallì completamente nella gestione dei profughi e degli sfollati, e nel rifornire la città di cibo, tanto che venne sostituito dall'ex prefetto Temistocle Testa, al quale venne dato il titolo di Commissario ai trasporti [Osti Guerrazzi 2018, 135].

Anche a Torino, Salerno diede prova di scarso "polso". Come scrive Nicola Adduci, Salerno venne promosso ad altro incarico dopo solo quattro mesi a Torino.

Sulla decisione pesano molti elementi: l'alto funzionario è certamente apparso inadeguato per la realtà torinese, a tratti addirittura succube di Solaro [il Federale] [...] e comunque scarsamente considerato dalle altre autorità [Adduci 2014, 255].

Non fu un caso, probabilmente, che a sostituirlo fosse chiamato Raffaele Manganiello, ex capo provincia di Firenze, considerato uno dei funzionari più duri e spietati del fascismo repubblicano, che non riuscì ad assumere la carica perché ucciso prima dai partigiani.

Il capo della provincia di Milano, Piero Parini, rappresenta un caso piuttosto simile. Anche Parini, infatti, era stato un combattente della Prima guerra mondiale, 
passato poi nelle fila fasciste come giornalista. Durante il regime aveva intrapreso la carriera diplomatica. Faceva parte dell'entourage di Dino Grandi, che lo volle nel suo ministero per avere funzionari di provata fede fascista. Diventò direttore generale della Direzione per gli italiani all'estero, ruolo che ricoprì fino alla guerra d'Etiopia, nella quale servì a capo di una legione di camicie nere. Collocato a riposo nel 1937 per dissidi con Galeazzo Ciano, fu poi riammesso in servizio come commissario per gli affari civili delle isole Jonie. Dopo l'armistizio, fu contattato da Francesco Maria Barracu che lo volle come capo della provincia e podestà di Milano [Dizionario Biografico 2014] ${ }^{20}$.

Durante la sua gestione della Prefettura, durata fino all'agosto del 1944, tentò di fare appello al patriottismo ambrosiano, in una prospettiva di superamento della guerra civile in nome di un nazionalismo conservatore e apartitico.

In questa congiuntura è difficile che l'esile patriottismo ambrosiano possa reggere a lungo. Eppure Parini si impegna a costruirlo, difenderlo, renderlo accetto ai moderati, popolarizzarlo presso chiunque possa apparire in qualche modo partecipe di questa prospettiva, fino a diventare lui stesso, nell'autunno-inverno 1944-45, uno dei paladini del socialismo nazionale della repubblica [Ganapini 1999, 321].

Le sue dimissioni arrivarono per protesta dopo l'eccidio di piazzale Loreto ${ }^{21}$, ma furono la conseguenza, probabilmente, di un dissenso notevole nei confronti della politica del fascismo più radicale. Secondo alcune testimonianze presentate nel processo a suo carico, dibattuto presso la Corte di Assise straordinaria di Milano nell'ottobre del 1945, avrebbe fornito aiuto a partigiani ed ebrei ${ }^{2}$. La sua opposizione alle deportazioni di ebrei si evince anche da altre testimonianze e documenti. Secondo la testimonianza rilasciata dal segretario generale del Comune al processo tenutosi a Milano, Parini cercò di opporsi a qualunque tentativo di intromissione da parte dei tedeschi ${ }^{23}$. Sempre secondo questa testimonianza, subito dopo l'occupazione fece nascondere i dati del censimento degli ebrei del 1938 e quando un impiegato del Comune cercò di recuperarli, su incarico dell'Ufficio politico investigativo (Upi) della Gnr, lo fece cacciare. Quando nel gennaio 1944 i tedeschi richiesero la consegna degli ebrei arrestati secondo l'ordine di polizia

\footnotetext{
20 Cfr. http://www.treccani.it/enciclopedia/piero-parini_Dizionario-Biografico/

21 Cfr. http://www.straginazifasciste.it/?page_id=38\&id_strage=1623

22 Archivio di stato di Milano (Asmi), Corte di Assise Speciale, b. 3, sentenza contro Parini Piero e Bettini Alberto. Secondo Carlo Silvestri Parini avrebbe addirittura nascosto degli ebrei nei sotterranei della Prefettura, ma della testimonianza dell'awocato Del Vecchio, che si sarebbe salvato proprio grazie all'intervento di Parini, non si hanno notizie nella sentenza qui citata.
}

23 Ibidem 
n. 5, Parini scrisse a Buffarini Guidi che non era riuscito a trovare un accordo con i nazisti. Probabilmente aveva cercato di evitare di consegnare gli ebrei rinchiusi a San Vittore, ma le autorità germaniche non avevano gradito ed avevano risposto che avrebbero chiesto ulteriori disposizioni ai loro superiori ${ }^{24}$.

Dopo aver dato le dimissioni, Parini aderì al Raggruppamento socialista repubblicano di Edmondo Cione, che però ebbe vita breve e nessuna influenza. Condannato per collaborazionismo dalla Corte di Assise straordinaria di Milano a 8 anni di detenzione, dopo un lungo iter processuale venne infine riabilitato.

Di Dino Fantozzi non si conosce invece praticamente nulla prima del 1939, quando diventò consigliere nazionale. Era stato anche commissario straordinario del comune di Acireale, cosa che fa pensare ad una carriera interna all'amministrazione statale [Pagano 2010, 89]. Il 14 gennaio 1944 diventò capo della provincia di Bologna, una provincia estremamente difficile dove la Federazione era in mano a personaggi tra i più radicali, quali Pietro Torri e Franz Pagliani. A Bologna operava la Compagnia autonoma speciale (Cas), reparto indipendente della Polizia comandato dal tristemente famoso Renato Tartarotti. La Cas aveva installato il suo quartier generale a Villa Campanati, una delle tante "ville tristi" che contrassegnarono la vita della Repubblica sociale. La prassi investigativa del reparto, secondo la sentenza della Corte di Assise straordinaria di Bologna, era la tortura che si svolgeva con

maltrattamenti che si attuavano mediante legature, a lungo protratte, degli inquisiti, su di un tavolo, con la testa e le estremità volte verso terra, battiture, scottature con ferro da stiro, olio bollente, cenere calda e bracia, strappamento di peli, applicazione ai piedi di corrente elettrica, senza alcun riguardo neppure al sesso e del pudore delle giovani patriote arrestate, brutalmente offese con atti abominevoli $i^{25}$.

La situazione, con l'arrivo della primavera, diventò sempre più difficile, dato l'espandersi della Resistenza. In luglio, scrive Luciano Bergonzini

fu tutto un susseguirsi di azioni armate in varie parti della città, con seguito di fucilazioni ed esecuzioni sommarie.

[...] Il 23 agosto in uno scontro in pieno centro con un milite che si accingeva ad arrestarlo, Stenio Polischi, 21 anni, uccise il milite ma non riuscì a sfuggire alla cattura. Trasportato a «Villa Triste» fu torturato da Tartarotti e dai militi Molmenti, D'Urdo e Rigon che lo martirizzarono con tizzoni ardenti, lo accecarono con spilloni negli occhi, poi lo trascinarono, bendato e ormai senza vita, in via Venezian per il lugubre rito dell'impiccagione pubblica [Bergonzini 1998, 111-112].

24 Acs, Ministero dell'Interno, Direzione Generale di Pubblica sicurezza, Divisione affari generali e riservati, A5G (II Guerra mondiale), b. 151, Telegramma di Piero Parini al Ministero dell'Interno del 27 gennaio 1944.

25 Acs, Ministero di Grazia e Giustizia, Grazie, Collaborazionisti, b. 23. Cfr. Franzinelli 2018, 93-100. 
È in un contesto di violenza radicale, priva di qualsiasi freno, che si trovò ad operare Fantozzi, il quale tentò di frenare l'escalation della guerra civile. Fantozzi, come d'altronde anche il generale Von Senger und Etterlin, era evidentemente un pragmatico, che capiva come questa strategia repressiva avrebbe portato, sul lungo periodo, solo "acqua al mulino" della Resistenza. Quando si trattò di gestire la famosa lista Jacchia, ovvero una lista di antifascisti che sarebbe stata trovata in possesso a Mario Jacchia al momento del suo arresto e che avrebbe portato alla fucilazione di alcuni di essi, Fantozzi si oppose al suo utilizzo a scopi terroristici, dicendo che si trattava di un elenco incompleto e sbagliato. Ma soprattutto Fantozzi si schierò con Von Senger und Etterlin nella sua aspra critica della Brigata nera, colpevole, in sintesi, di turbare l'ordine e l'opinione pubblica con il suo comportamento indisciplinato e violento [Ganapini 1999, 50; Bergonzini 1998, 276-282].

Questi brevi percorsi biografici presentano delle evidenti contraddizioni. Se si paragonano un Parini ed un Salerno a Vezzalini e a Grazioli, le differenze sono abbastanza evidenti. In generale si tratta di fascisti convinti [Stefanori 2017, 192], dato più che evidente che si evince dal fatto che avevano aderito alla Rsi e che, in maniera altrettanto evidente, godevano della fiducia, se non di Mussolini, almeno del ministro Buffarini Guidi. È vero che nella Rsi furono "arruolati", in mancanza di alternative, anche personaggi non particolarmente efficienti o di spicco, ma è assai improbabile che, se anche non straordinariamente capaci, essi rappresentassero comunque persone affidabili dal punto di vista politico. Tuttavia il comportamento di questi capi provincia differì moltissimo. Da una parte, dei radicali, particolarmente decisi, se non feroci, nell'applicare le direttive repressive del governo, andando a volte anche oltre gli ordini; dall'altra, dei funzionari che si limitarono ad applicare le leggi, non dando prova di particolare iniziativa. Non si tratta qui, forse, soltanto della nota suddivisione tra moderati e radicali. Una specifica opinione politica poco consona al momento si può notare soltanto in Parini, che aderì al Raggruppamento di Cione e quindi aveva una sua autonoma visione del futuro della Repubblica. Salerno, così come Renato Celio, capo della provincia di Asti, erano semplicemente dei funzionari, dei servitori dello Stato fascista che però, forse per non compromettersi troppo, oppure perché disapprovavano l'estrema violenza di Brigate nere o organismi militari consimili, tentarono di limitare i danni e di applicare le leggi in maniera più o meno razionale. I radicali, invece, erano dei nazifascisti convinti, ovvero erano talmente immedesimati nel progetto di un Nuovo ordine europeo con a capo Hitler e in subordine Mussolini, da impegnarsi fino alla fine in una guerra che sapevano andare verso una conclusione ingloriosa utilizzando mezzi estremi. Da una parte, quindi, dei 
burocrati, anche se fascisti; dall'altra dei soldati politici, dei convinti sostenitori della guerra dell'Asse e dei suoi scopi.

Queste differenze si evidenziano con chiarezza nella delicata questione delle deportazioni degli ebrei, alla quale i capi provincia non risposero in maniera univoca. Ad esempio il capo della provincia di Asti, Renato Celio, evitò di prendere iniziative personali e cercò, per quanto possibile, di opporsi alle deportazioni di donne, di malati e di vecchi [Fasano, Renosio 2010, 55]. Se pure collaborò nella deportazione dei soggetti previsti dalle leggi emanate dalla Rsi, non dimostrò un grande zelo nella ricerca dei fuggiaschi. Invece il capo della provincia di Grosseto, Alceo Ercolani, si dimostrò di tutt'altra tempra, anticipando addirittura l'ordine di polizia n. 5 e istituendo il campo di Roccatederighi due giorni prima dell'emanazione dell'ordine di creare i campi provinciali [Stefanori 2017, 72].

L'esempio della persecuzione antiebraica è particolarmente significativo anche perché le leggi della Rsi erano, forse volutamente, lacunose e lasciavano spazio alla interpretazione dei singoli. Così, quando tra il gennaio e il febbraio 1944 arrivarono forti pressioni naziste per la cessione degli ebrei internati e il loro trasferimento al campo di Fossoli (per il conseguente trasporto ad Auschwitz), e mentre Buffarini Guidi e Mussolini non davano istruzioni precise, i capi provincia furono costretti a prendere delle decisioni in proprio. Posti di fronte alla propria coscienza, tra i capi provincia vi fu chi, come Vezzalini, apparentemente non ebbe dubbi e consegnò immediatamente i "suoi" ebrei ${ }^{26}$; e chi, come Parini, tentò almeno di ritardarne la consegna.

Le stesse diversità si ritrovano anche ai gradi più bassi dell'amministrazione. A Roma il reggente della Questura, Luigi Roselli, diede scarsa prova di efficienza, soprattutto riguardo alla persecuzione degli ebrei [Osti Guerrazzi 2017, 155]. Fu sostituito dal questore Pietro Caruso, che non appena arrivato in sede diede ordine di intensificare la sorveglianza e gli arresti degli ebrei [Picciotto 1979].

Come si possono interpretare queste differenze? Quali motivi portarono funzionari dichiaratamente fascisti a applicare le leggi e i provvedimenti in maniera così diversa?

Sicuramente, come scrive Stefanori, i motivi umanitari giocarono un loro ruolo. Oggi siamo abbastanza abituati a pensare che nell'Europa nazista inviare donne e bambini nei campi di sterminio fosse una cosa "normale", ma normale non lo era affatto. I leader nazisti impiegarono anni a convincere i loro "uomini comuni" a uccidere a sangue freddo intere comunità, e comunque il timore dei contraccolpi

Acs, Ministero dell'Interno, Direzione generale di Pubblica sicurezza, Divisione affari generali e riservati, A5G (II Guerra mondiale), b. 151, Telegramma di Vezzalini al Ministero dell'Interno dell'11 febbraio 1944. 
psicologici sul personale degli Einsatzgruppen portò i vertici dell'Rsha (Reichssicherheitshauptamt) a creare il sistema più asettico dello sterminio industriale, di cui le camere a gas furono l'apice. In Jugoslavia e in Francia i soldati italiani, davanti ai massacri o al timore delle deportazioni, si opposero in larga maggioranza ai nazisti. Non si può quindi escludere a priori che anche alcuni funzionari della Rsi, che non erano stati formati e abituati ad una violenza di questo tipo, avessero dei dubbi e tentassero quantomeno di rallentare le deportazioni. Anche il conflitto di competenze con la polizia nazista può avere giocato un ruolo nel rifiuto di obbedire ad ordini che, a partire da gennaio 1944, provenivano dai comandi tedeschi, a cui in teoria $i$ capi provincia non erano subordinati.

Altri invece non ebbero scrupoli o dubbi particolari. E questa differenza si può tentare di spiegare con le differenti esperienze precedenti. Personaggi come Vezzalini o Grazioli erano non solo dei funzionari statali, ma anche e soprattutto dei fascisti che avevano combattuto quasi tutte le guerre del regime. Provenivano dalle fila del Partito e, soprattutto, avevano avuto delle esperienze altamente brutalizzanti, che li avevano abituati alla violenza, anche quella più estrema. Non erano soltanto dei servitori dello Stato, facevano parte della categoria dell' "uomo nuovo" fascista, erano dei guerrieri politici abituati a combattere e a distruggere i nemici dell'ideale senza porsi alcun dubbio. Sarebbe necessaria una analisi più approfondita delle biografie di questi personaggi e tuttavia le esperienze di un Grazioli o di un Basile appaiono sufficientemente rappresentative di una generazione che aveva svolto l'intera carriera nel Partito, che aveva vissuto il crollo del regime come una tragedia epocale, personale e collettiva e che, dopo 1'8 settembre, tornò al potere con una carica di rabbia e di violenza che la spinse a mettere in atto ogni mezzo, pur di far trionfare la causa del fascismo. Con una mentalità del genere, con queste esperienze alle spalle, consegnare alcuni anziani ebrei ai nazisti non rappresentava, molto probabilmente, un crimine o un atto riprovevole, ma semplicemente un atto dovuto nel quadro della guerra civile europea. Gli ebrei erano parte di quel "mondo di nemici" che andava sterminato, se si voleva realizzare il progetto di una Europa nazifascista.

Le scelte di Mussolini, inoltre, evidenziano la radicalizzazione del conflitto e il ruolo che il dittatore ebbe nella brutalizzazione della guerra civile. Era Mussolini, dalla sua scrivania, a decidere le carriere dei funzionari, era lui che dava visibilità e potere attraverso le udienze ai capi locali. La progressiva eliminazione dei capi provincia meno violenti e la progressiva erosione dei funzionari statali tradizionali in favore dei vari capi banda furono anche il risultato di decisioni prese proprio dal duce che - come vedremo - concesse spazio e potere a personaggi come Franco Colombo e Fiorentini. 


\section{Poliarchia in provincia e contraddizioni del potere centrale}

Data l'incapacità della Rsi, a tutti i livelli, di riportare legge ed ordine e di stroncare la Resistenza, Mussolini dovette rassegnarsi a lasciare spazio e potere anche a quei gruppi autonomi invisi ai prefetti, che ricordavano molto da vicino le compagnie di ventura rinascimentali, anche se tentava di stringere la disciplina cercando di ricostituire il sistema delle reti locali di potere attorno ai capi provincia, che assursero ad un ruolo non più solo eminentemente di cinghia di trasmissione del potere centrale, ma di veri e propri rappresentanti quasi personali di Mussolini nelle province. Tuttavia queste contraddizioni nella politica mussoliniana, che da una parte si affidava ai capi provincia e dall'altra ne minava l'autorità dando potere alle unità militari e polizie autonome o semi autonome, creò non pochi problemi.

L'incessante girandola di capi provincia era anche il sintomo dell'ingovernabilità di alcune zone, dove chi comandava erano in realtà i tedeschi (nelle città e nelle zone pianeggianti), appoggiati dalle varie milizie speciali e dalle Brigate nere (a partire dall'estate del 1944), con risultati estremamente negativi per quanto riguarda il prestigio del potere centrale. Nelle montagne, invece, erano i partigiani a fare "il bello e cattivo tempo" mentre la Guardia nazionale repubblicana, sempre in piena crisi per la mancanza di armi, equipaggiamento e personale addestrato e motivato, ritirava i propri presidi per non finire nelle mani della Resistenza. I rapporti di un ispettore generale di Pubblica sicurezza, dell'estate del 1944 relativi al Piemonte, restituiscono con particolare chiarezza il tracollo dell'autorità statuale in questo periodo.

La situazione generale nelle varie provincie di questo Settore permane grave, non essendosi verificato finora nessun fatto nuovo, che possa tranquillizzare la popolazione. Continuano le violenze, gli atti di rappresaglia, i rastrellamenti, le rapine, ecc. Nelle vallate del Cuneese, come viene riferito a parte nella presente relazione, i partigiani si sono sostituiti completamente agli organi dello Stato. Anche nel campo annonario vi sono moltissime lamentele per il persistere della «borsa nera» e per la confezione del pane, che, in questo periodo di congiuntura, è pessimo per essere anche lavorato senza lievito e sale ${ }^{27}$.

Tenendo conto che la Prefettura era la responsabile sia dell'ordine pubblico, che della sorveglianza sulla distribuzione del cibo, nonché della soppressione della borsa nera, queste poche righe riflettono un fallimento totale delle autorità locali, e in special modo dell'autorità prefettizia.

Acs, Ministero dell'Interno, Segreteria particolare del Capo della polizia Rsi, b. 63, Rapporto dall'Ispettorato speciale generale di Polizia del 30 agosto 1944. 
In ottobre lo stesso alto funzionario di Pubblica sicurezza rincarava la dose, osservando che:

L'indisciplina e gli abusi da parte di elementi delle Forse Armate Repubblicane, e particolarmente da militi della $\mathrm{X}^{\circ}$ Flottiglia MAS sono sempre più frequenti e palesi e ciò aumenta quello stato d'animo avverso e di sfiducia che già regna nella popolazione, alienandosi completamente quelle poche simpatie che si erano create. Sempre più frequenti sono gli incidenti provocati da parte di elementi della $\mathrm{X}^{\circ}$ Flottiglia Mas nei riguardi della Polizia, come da relazione a parte.

La situazione annonaria è grave e vivaci lamentele si levano dalla popolazione contro le Autorità per gli approvvigionamenti di combustibili e per il riscaldamento del prossimo inverno ${ }^{28}$.

Se a Torino era la X Mas a rendersi particolarmente odiosa, a Milano erano la Brigata nera e la Legione autonoma Ettore Muti a rendersi tristemente famose per l'indisciplina e la violenza gratuite. Il capo provincia Mario Bassi, il 16 aprile 1945, era costretto a chiedere chiarimenti a Enzo Costa (Federale e comandante della Brigata nera), perché in provincia da un camion della Brigata nera erano partiti colpi di fucile, che sparati senza alcun motivo apparente avevano causato la morte di una bambina di due anni ${ }^{29}$.

La totale anarchia che vigeva nelle provincie venne invano contrastata prima da Tullio Tamburini, e poi da Renzo Montagna, i capi della Polizia repubblicana, che tentarono di unificare le forze di polizia e soprattutto di stroncare il fenomeno dei vari reparti speciali o delle legioni autonome. Tamburini, nel novembre del 1944, scrisse a Buffarini Guidi sottolineando proprio i danni di questa frammentazione delle forze che si arrogavano i poteri di polizia giudiziaria:

Gli inconvenienti di avere troppi organismi di polizia che arrestano, perquisiscono, fucilano etc. sono noti ed altrettanto noti sono i danni causati da servizi di informazione non controllati ed in genere poco seri ${ }^{30}$.

La poliarchia che si era instaurata nelle provincie costrinse le autorità centrali a tentare di intervenire per ridare prestigio alle figure istituzionali dei capi provincia. Nel febbraio del 1944, in una riunione dei capi provincia di Piemonte, Emilia, Lombardia e Veneto, tutti i presenti lamentarono «lo stato di incertezza e di confusione che si verifica nel campo della polizia» ${ }^{31}$ [Ganapini 1999, 282]. Un

\footnotetext{
28 Ivi, Rapporto dall'Ispettorato speciale generale di Polizia del 30 ottobre 1944.

29 Asmi, Prefettura, Gabinetto, b. 364, Lettera di Mario Bassi al comandante della Brigata nera del 16 aprile 1945.

30 Acs, Ministero dell'Interno, Segreteria particolare del Capo della polizia Rsi, b. 33, Lettera di Tamburini a Buffarini Guidi del 14 settembre 1944.

31 Acs, Segreteria particolare del Duce Rsi, Carteggio riservato, b. 79.
} 
anno dopo, nel febbraio del 1945 Zerbino, allora ministro degli interni, emanò una circolare a tutti gli uffici dipendenti nella quale si leggeva:

Il Capo della Provincia è il solo responsabile politico dell'ordine pubblico nell'ambito provinciale. Il Questore è il solo responsabile tecnico dell'ordine pubblico nell'ambito provinciale. La Guardia Nazionale Repubblicana con l'annesso servizio UPI, per quanto attiene ad operazioni di polizia, dipende perifericamente dal Capo della Provincia e dal Questore. Nessun reparto armato può compiere operazioni di polizia quando non vi sia autorizzato e per ragioni di carattere eccezionale dagli organi competente dal governo [Ganapini 1999, 295] ${ }^{32}$.

Un ultimo tentativo per accentrare il potere a livello regionale venne fatto nel 1945, con la creazione della figura del "Commissario straordinario" per alcune regioni. Ad esempio l'ex prefetto di Torino, Emilio Grazioli, venne nominato Commissario straordinario per il Piemonte, con risultati che, alla luce delle poche fonti disponibili, è decisamente difficile stabilire. Dato l'avanzato stato di disfacimento della Repubblica, nei suoi ultimi mesi di agonia è comunque possibile ipotizzare che questa ultima misura abbia segnato più la disperazione di Mussolini, che un provvedimento realmente efficace. Bande, reparti speciali e gruppi autonomi, infatti, continueranno a infestare le più importanti città del Nord fino alla fine della Repubblica.

Il quadro della situazione delineato in queste pagine è piuttosto chiaro. Da una parte, il potere centrale, e quindi Mussolini, che tentava invano di imporre un minimo di controllo sulle autorità locali attraverso i prefetti, di alcuni dei quali però evidentemente non si fidava e sostituiva o faceva ruotare nelle varie sedi, minandone così il potere e il prestigio; dall'altra, una serie infinita di ras e capi locali che minavano a loro volta il potere dei capi provincia, arrogandosi ruoli e poteri che non spettavano loro ${ }^{33}$. Un altro problema proveniva nuovamente dalle iniziative dello stesso Mussolini che, contraddicendosi, dava lustro e prestigio alle varie bande ricevendo i loro dirigenti a Gargnano o andando in visita ai loro quartieri generali, come fece a Milano nel dicembre 1944 quando si recò in visita alla sede della Legione autonoma Ettore Muti. A tutto questo si aggiungeva l'ambiguo comportamento del ministro dell'Interno Guido Buffarini Guidi, che

Tassative disposizioni del Ministero degli Interni, 2 marzo 1945, in Acs, GNR, b. 19. La circolare venne pubblicata anche sul "Corriere della Sera". Sul caos dovuto al proliferare delle polizie, si veda: Ganapini 1999, 275-295.

33 Sul ruolo centrale dei capi provincia nei rapporti di forza tra le varie istanze statali e parastatali nella Rsi, e in particolare in Emilia Romagna, si veda: Preti 1993, 305-316. 
spesso si appoggiava alle varie "polizie speciali", come ad esempio la "Banda Koch", che entravano direttamente in contrasto con la polizia "tradizionale" ${ }^{34}$. In conclusione si può dire che Mussolini, nonostante tutti i suoi tentativi di riaffermare il potere dello Stato attraverso la ricostruzione di una rete di istituzioni tradizionali, non riuscì, e in alcuni casi non volle, imporre l'autorità dei capi provincia e dovette piegarsi ad accettare l'emergere di bande e gruppi autonomi. Tale processo fu sicuramente dovuto all'influenza dei tedeschi, che gestivano le situazioni locali con enorme spregiudicatezza e ignoravano le gerarchie ufficiali della Repubblica, e alla radicalizzazione della guerra civile. Fu lo stesso Mussolini, in sintesi, sconcertato dall'inefficienza e dalla poca decisione di molti dei suoi funzionari tradizionali, a minarne il ruolo e l'autorità dando spazio e visibilità ad alcuni dei peggiori "banditi” della Rsi. Il sogno di una repubblica ordinata, di uno stato efficiente ed autorevole in grado di riaffermare la propria autorevolezza anche attraverso una gestione ordinata e legale della repressione, si infranse contro difficoltà oggettive ed insormontabili. Esasperato dal suo stesso fallimento, Mussolini decise di affidarsi anche a personaggi - tra i quali anche alcuni capi provincia - la cui brutalità e violenza non avevano nulla a che invidiare a quella dei più radicali nazisti.

Il presente contributo è una rielaborazione rivista ed ampliata della relazione presentata al convegno "I molti territori della Repubblica fascista. Amministrazione e società nella RSI", Ferrara 27/28 settembre 2017. A fronte dell'impossibilità di sottoporre il testo ad un processo di double blind peer-review, i curatori hanno optato per una revisione preliminare da parte del relativo discussant di sessione. 


\section{Bibliografia:}

Adduci N. 2014, Gli altri. Fascismo repubblicano e comunità nel torinese (1943-1945), Milano: Angeli.

Antonini S. 2007, La banda Spiotta e la brigata nera genovese Silvio Parodi. Una anatomia dei crimini fascisti. 1943-1945, Genova: De Ferrari.

Balugani R. 1999, La scia di sangue lasciata dai tupin (1943-1945), Modena: Sigem.

Bergonzini L. 1998, La svastica a Bologna. Settembre 1943 - aprile 1945, Bologna: il Mulino.

Caporale R. 2005, La Banda Carità: storia del Reparto servizi speciali, 1943-45, Lucca: S. Marco Litotipo.

Cuzzi M. 1998, L'occupazione italiana della Slovenia, Roma: Stato maggiore dell'Esercito, Ufficio Storico.

Deakin F. W. 1963, Storia della Repubblica di Salò, Torino: Einaudi.

Dizionario Biografico degli Italiani 2014, Vol. 81, Roma: Treccani.

Fasano N. e Renosio M. 2010, La deportazione dalla provincia di Asti, in Mantelli B. (ed) 2010, Il libro dei deportati, vol. II Deportati, deportatori, tempi, luoghi, Milano: Mursia.

Franzinelli M. 2018, Tortura. Storie dell'occupazione nazista e della guerra civile, Milano: Mondadori.

Ganapini L. 1999, La repubblica delle camicie nere, Milano: Garzanti.

Griner M. 2000, La “Banda Koch”. Il Reparto speciale di polizia 1943-1944, Torino: Bollati Boringhieri.

Griner M. 2004, La pupilla del duce. La legione mobile autonoma Ettore Muti, Torino: Bollati Boringhieri.

Kershaw I. 2000, Hitler e l'enigma del consenso, Bari-Roma: Laterza.

Klinkhammer L. 1993, L'occupazione tedesca in Italia. 1943-1945, Torino: Bollati Boringhieri.

Lupo S. 2000, Il fascismo. La politica in un regime totalitario, Roma: Donzelli.

Mayda G. 2002, Storia della deportazione dall'Italia, Torino: Bollati Boringhieri.

Osti Guerrazzi A. 2017, La persecuzione degli ebrei a Roma. Carnefici e vittime, in Haia Antonucci S. e Procaccia C. (eds) 2017, Dopo il 16 ottobre. Gli ebrei a Roma tra occupazione, resistenza, accoglienza e delazioni (1943-1944), Roma: Viella.

Osti Guerrazzi A. 2018, L'inferno sulla città santa. Roma 1940-1944, in Labanca N. (ed.) 2018, Città sotto le bombe. Per una storia delle vittime civili di guerra (1940-1945), Milano: Unicopli.

Pagano M.C. 2010, Il fascio e la croce. Clero e classi dirigenti ad Acireale tra le due guerre, Acireale: Tipografia ACI. 
Parisini R. 2005, Dal regime corporativo alla Repubblica sociale. Agricoltura e fascismo a Ferrara. 1928-1945, Ferrara: Gabriele Corbo Editore

Picciotto L. 1979, L'occupazione tedesca e gli ebrei di Roma, Roma: Carucci

Preti A. 1993, Assetto e rappresentazione del potere nella RSI. Le provincie emiliane, "Italia contemporanea", 191

Rossi S.G.S. 2005, Mussolini e il diplomatico. La vita e i diari di Serafino Mazzolini, un monarchico a Salò, Soveria Mannelli: Rubbettino

Savino E. 1937, La nazione operante, III edizione, Novara: De Agostini

Spampanato B. 1957, Contromemoriale, Roma: Cen

Stefanori M. 2017, Ordinaria amministrazione. Gli ebrei e la Repubblica sociale italiana, Bari-Roma: Laterza 